\title{
Stability analysis of second-order time accurate schemes for ALE-FEM
}

\author{
Luca Formaggia $^{\mathrm{a}, *}$, Fabio Nobile ${ }^{\mathrm{b}}$ \\ ${ }^{a}$ MOX, Mathematics Department, Politecnico di Milano, Milan, Italy \\ ${ }^{\mathrm{b}}$ ICES, The University of Texas at Austin, TX 78712, USA
}

Received 16 May 2003; received in revised form 29 August 2003; accepted 4 September 2003

\begin{abstract}
In this work we will introduce and analyze the Arbitrary Lagrangian Eulerian formulation for a model problem of a scalar advection-diffusion equation defined on a moving domain. Moving from the results illustrated in our previous work [J. Num. Math. 7 (1999) 105], we will consider first and second-order time advancing schemes and analyze how the movement of the domain might affect accuracy and stability properties of the numerical schemes with respect to their counterpart on fixed domains. Theoretical and numerical results will be presented, showing that stability properties are not, in general, preserved, while accuracy is maintained.
\end{abstract}

(C) 2004 Elsevier B.V. All rights reserved.

Keywords: Stability analysis; Arbitrary Lagrangian Eulerian formulation; Finite elements; Time advancing schemes

\section{Introduction}

One of the most used techniques for the numerical simulation of partial differential equations on moving domains is the so-called Arbitrary Lagrangian Eulerian (ALE) formulation. In this formulation the partial time derivative is expressed with respect to a reference fixed configuration. A special homeomorphic map, called the ALE map $\mathscr{A}_{t}: \Omega_{0} \rightarrow \Omega(t)$ associates, at each time $t$, a point in the current computational domain $\Omega(t)$ to a point in the reference domain $\Omega_{0}$.

In this way, the ODE system resulting after space discretization actually describes the evolution of the solution along trajectories that are at all times contained in the computational domain. The ALE mapping is somehow arbitrary, apart from the requirement of conforming to the evolution of the domain boundary, which is either a given data or the result of the coupling with other differential models. The latter is the case, for instance, when treating fluid-structure interaction problems where the position of the fluid domain

\footnotetext{
${ }^{*}$ Corresponding author.

E-mail address: luca.formaggia@mate.polimi.it (L. Formaggia).
} 
boundary is provided through the interaction with a mechanical model. The map of the boundary $\partial \Omega_{0}$ of the reference domain has then to provide, at all $t$, the boundary of the current configuration $\Omega(t)$.

In a numerical simulation we are concerned with the evolution of the discrete domain, typically built as the assembly of the elements of a computational grid. The discrete ALE map describes the evolution of the grid during the domain movement. It is indeed at discrete level that the advantage of the ALE formulation emerges, as in an ALE setting the time advancing scheme provides directly the evolution of the unknowns at mesh nodes, and thus, in a classic finite element setting, that of the degrees of freedom of the discrete problem at hand.

Several issues have however to be addressed when using the ALE formulation in a finite element context. What is the minimal regularity for the ALE mapping to ensure the well-posedness of the differential problem in the ALE formulation? What type of finite element discretization is appropriate for the discrete ALE mapping in relation to the finite element space chosen for the numerical solution? How the stability of the numerical scheme is affected by the mesh movement? These are some of the questions addressed by the authors in [4] for a model advection-diffusion problem. In particular, in this reference it is shown how the fulfillment of the so called Geometric Conservation Law is a sufficient condition for the Backward Euler implicit scheme to be unconditionally stable. In fact the ALE formulation introduces an additional advection term, which is related to the grid velocity (i.e. the time derivative of the discrete ALE mapping). The stability of the time advancing scheme may be affected by this term, which is normally difficult to control.

The Geometric Conservation Law (GCL) was originally introduced in [12] and has been recently dealt in more details by Fahrat and coworkers. More specifically its significance in a finite volume context and for aero-elasticity problems has been tackled in [6,9], while in [7] a procedure is given to build schemes for unstructured dynamic meshes that show (by numerical experimentation) a second-order time accuracy. The GCL condition is, in fact, a request of strong consistency of the discrete problem with respect to constant solutions: the numerical scheme must be able to exactly represent constant solutions. In [4] its significance for finite element formulations on moving grids is further clarified by establishing a clear link with the degree of exactness of the time advancing scheme. In [3] it is shown how for a scalar non-linear hyperbolic conservation law discretized by means of monotone numerical fluxes, the satisfaction of GCL is a sufficient and necessary condition for the numerical scheme to preserve a maximum principle when applied on moving meshes. However, in a later work [5], the same authors, by means of truncation error arguments, show that the GCL is not a necessary condition to obtain a (formally) second-order time accurate scheme. In that work finite volume schemes are considered, applied to aero-elastic problems.

In the present work, we reach the same conclusions by considering the finite element discretization of a model scalar advection-diffusion problem. We will consider first and second-order time advancing schemes and analyze how the domain movement might affect the stability and accuracy properties of the numerical schemes with respect to their counterpart on fixed domains and what role does the GCL play with this respect. The numerical tests presented in Section 5 show that time accuracy of the numerical schemes is preserved irrespectively to the satisfaction of the GCL, provided the domain movement is suitably interpolated. Conversely, we will show, both theoretically and numerically, that the stability of the most known second-order unconditionally stable (on fixed grids) schemes is affected by the domain movement, whether or not the GCL is satisfied, with the only exception of the backward Euler case, as already shown in [4]. This in practice means that a condition on the time step has to be imposed to ensure stability. However, although the results presented in Section 5 show a spurious "energy production" as a consequence of the moving mesh, we were actually unable to find a test case where the simulation would blow-up. This probably shows that in practice (at least for linear problems) the instability induced by the domain movement is mild.

The paper is organized as follows. In Section 2 we introduce the finite element formulation in ALE frame. In Section 3 we recall, for the sake of completeness, the principal results obtained in our previous 
work. In Section 4 we derive stability inequalities for two second-order schemes (namely Crank-Nicolson and second-order backward finite difference scheme) and prove a conditional stability for both of them. In Section 5 we analyze numerically the stability and accuracy of the different schemes. Finally, in Section 6 we draw some conclusions.

\section{ALE formulation and finite element approximation}

Let us consider the following model problem defined on a moving domain $\Omega_{t} \subset \mathbb{R}^{d}, t \in I \equiv\left(t_{0}, T\right)$ :

$$
\begin{array}{ll}
\frac{\partial u}{\partial t}+\nabla \cdot(\boldsymbol{\beta} u)-\mu \Delta u=f & \text { in } \Omega_{t}, \quad t \in I \\
u=u_{0} & \text { in } \Omega_{0}, \quad t=t_{0} \\
u=u_{\mathrm{D}} & \text { on } \partial \Omega_{t}, \quad t \in I,
\end{array}
$$

where $\boldsymbol{\beta}$ is a convection velocity, which is assumed to satisfy $\boldsymbol{\nabla} \cdot \boldsymbol{\beta}=0, \mu$ a constant diffusivity, $\Delta$ indicates the Laplacian operator and $u_{\mathrm{D}}$ is an assigned boundary condition of Dirichlet type.

For this problem, and for the homogeneous case $u_{\mathrm{D}}=0$, an a priori energy inequality can be easily obtained (see for instance [4]), and it reads

$$
\|u(t)\|_{L_{2}\left(\Omega_{t}\right)}^{2}+\mu \int_{t_{0}}^{t}\|\nabla u(s)\|_{L_{2}\left(\Omega_{s}\right)}^{2} \mathrm{~d} s \leqslant\left\|u\left(t_{0}\right)\right\|_{L_{2}\left(\Omega_{t_{0}}\right)}^{2}+\frac{\left(1+C_{\Omega}\right)}{\mu} \int_{t_{0}}^{t}\|f(s)\|_{H^{-1}\left(\Omega_{s}\right)}^{2} \mathrm{~d} s, \quad \forall t \in I,
$$

where we have denoted by $C_{\Omega}$ the constant appearing in the Poincaré inequality:

$$
\|v\|_{L_{2}\left(\Omega_{t}\right)}^{2} \leqslant C_{\Omega}\|\nabla v\|_{L_{2}\left(\Omega_{t}\right)}^{2}, \quad \forall v \in H_{0}^{1}\left(\Omega_{t}\right)
$$

The Sobolev spaces $H^{1}(\Omega)$ and $H^{-1}(\Omega)$ and the corresponding norms are defined in the standard way (see, for instance, $[2,11]$ ).

The Arbitrary Lagrangian Eulerian (ALE) formulation recasts the original problem (1) on a reference fixed configuration, say $\Omega_{0}$ (this might be, for instance, the domain configuration at time $t=t_{0}$ ).

Let $\mathscr{A}_{t}$ be a family of mappings, hereafter called ALE mappings, which at each $t \in I$ associate a point $\mathbf{Y}$ of the reference configuration $\Omega_{0}$ to a point $\mathbf{x}$ on the current domain configuration $\Omega_{t}$ :

$$
\mathscr{A}_{t}: \Omega_{0} \subset \mathbb{R}^{d} \rightarrow \Omega_{t} \subset \mathbb{R}^{d}, \quad \mathbf{x}(\mathbf{Y}, t)=\mathscr{A}_{t}(\mathbf{Y}) .
$$

We assume $\mathscr{A}_{t}$ to be an homeomorphism, that is $\mathscr{A}_{t} \in C^{0}\left(\overline{\Omega_{0}}\right)$ is invertible with inverse $\mathscr{A}_{t}^{-1} \in C^{0}\left(\overline{\Omega_{t}}\right)$. Furthermore, we assume that the application $t \rightarrow \mathbf{x}(\cdot, t)$ is differentiable almost everywhere in $\left[t_{0}, T\right]$. We may note that the mapping is in fact rather arbitrary, apart from the requirement that, for all $t \in I$, $\mathscr{A}_{t}\left(\partial \Omega_{0}\right)=\partial \Omega_{t}$.

We name $\mathbf{Y} \in \Omega_{0}$ the $A L E$ coordinate while $\mathbf{x}=\mathbf{x}(\mathbf{Y}, t)$ will be addressed as the spatial (or Eulerian) coordinate. For the sake of notation, we put $\Omega_{t} \times I=\left\{(\mathbf{x}, t) \mid \mathbf{x} \in \Omega_{t}, t \in I\right\}$.

Let $f: \Omega_{t} \times I \rightarrow \mathbb{R}$. We will indicate with $\hat{f}:=f \circ \mathscr{A}_{t}$ the corresponding function on the ALE frame, i.e.

$$
\hat{f}: \Omega_{0} \times I \rightarrow \mathbb{R}, \quad \hat{f}(\mathbf{Y}, t)=f\left(\mathscr{A}_{t}(\mathbf{Y}), t\right) .
$$

Note that the composition operator applies only to the spatial variables, being the time variable $t$ left unchanged by the mapping. We will adopt the symbol $\left.\frac{\partial f}{\partial t}\right|_{\mathbf{Y}}$ to indicate the ALE time derivative, defined as

$$
\left.\frac{\partial f}{\partial t}\right|_{\mathbf{Y}}: \Omega_{t} \times I \rightarrow \mathbb{R},\left.\quad \frac{\partial f}{\partial t}\right|_{\mathbf{Y}}(\mathbf{x}, t)=\frac{\partial \hat{f}}{\partial t}(\mathbf{Y}, t), \quad \mathbf{Y}=\mathscr{A}_{t}^{-1}(\mathbf{x}) .
$$


For analogy, from now on we will use $\left.\frac{\partial f}{\partial t}\right|_{\mathbf{x}}$ for the partial time derivative in the Eulerian frame. Finally, we define the domain velocity $\hat{\mathbf{w}}$ as

$$
\hat{\mathbf{w}}(\mathbf{Y}, t)=\left.\frac{\partial \mathbf{x}}{\partial t}\right|_{\mathbf{Y}}(\mathbf{Y}, t)
$$

and indicate with $\mathbf{w}$ the corresponding function in $\Omega_{t} \times I$. We have that

$$
\left.\frac{\partial f}{\partial t}\right|_{\mathbf{Y}}=\left.\frac{\partial f}{\partial t}\right|_{\mathbf{x}}+\left.\frac{\partial \mathbf{x}}{\partial t}\right|_{\mathbf{Y}} \cdot \nabla_{\mathbf{x}} f=\left.\frac{\partial f}{\partial t}\right|_{\mathbf{x}}+\mathbf{w} \cdot \nabla_{\mathbf{x}} f .
$$

The model problem (1) in the ALE frame then reads [4]

$$
\begin{array}{ll}
\left.\frac{\partial u}{\partial t}\right|_{\mathbf{Y}}-\mathbf{w} \cdot \nabla_{\mathbf{x}} u+\nabla_{\mathbf{x}} \cdot(\boldsymbol{\beta} u)-\mu \Delta_{\mathbf{x}} u=f & \text { for } \mathbf{x} \in \Omega_{t}, \quad t \in I \\
u=u_{0} & \text { for } \mathbf{x} \in \Omega_{0}, \quad t=t_{0} \\
u=u_{\mathrm{D}} & \text { for } \mathbf{x} \in \partial \Omega_{t}, \quad t \in I,
\end{array}
$$

where we have indicated the gradient, divergence and Laplace operators with $\nabla_{\mathbf{x}}, \nabla_{\mathbf{x}}$. and $\Delta_{\mathbf{x}}$, respectively, to underline that the space derivatives are taken with respect to the Eulerian coordinate $\mathbf{x}$. Formulation (6) will be referred to as the non-conservative ALE formulation.

Whenever the conservative properties of the problem are important, a conservative formulation may be desirable. This is readily obtained by noting that [1]

$$
\left.\frac{\partial J_{\mathscr{A}_{t}}}{\partial t}\right|_{\mathbf{Y}}=J_{\mathscr{A}_{t}} \nabla_{\mathbf{x}} \cdot \mathbf{w}
$$

where $J_{\mathscr{A}_{t}}$ is the Jacobian of the ALE mapping

$$
J_{\mathscr{A}_{t}}=\operatorname{det}\left(\mathbf{J}_{\mathscr{A}_{t}}\right), \quad \text { and } \quad \mathbf{J}_{\mathscr{A}_{t}}=\frac{\partial \mathbf{x}}{\partial \mathbf{Y}} .
$$

We have, then,

$$
\left.\frac{1}{J_{\mathscr{A}_{t}}} \frac{\partial\left(J_{\mathscr{A}_{t}} u\right)}{\partial t}\right|_{\mathbf{Y}}=\left.\frac{\partial u}{\partial t}\right|_{\mathbf{Y}}+\left.\frac{1}{J_{\mathscr{A} t}} \frac{\partial J_{\mathscr{A}_{t}}}{\partial t}\right|_{\mathbf{Y}} u=\left.\frac{\partial u}{\partial t}\right|_{\mathbf{Y}}+u \nabla_{\mathbf{X}} \cdot \mathbf{W}
$$

and the differential equation in (1) can be rewritten in the following conservative ALE form

$$
\left.\frac{1}{J_{\mathscr{A}_{t}}} \frac{\partial J_{\mathscr{A}_{t}} u}{\partial t}\right|_{\mathbf{Y}}+\nabla_{\mathbf{x}} \cdot\left[(\boldsymbol{\beta}-\mathbf{w}) u-\mu \nabla_{\mathbf{x}} u\right]=f \quad \text { for } \mathbf{x} \in \Omega_{t}, \quad t \in I .
$$

Remark 1. We may also introduce the mapping $\mathscr{A}_{r}^{s}: \Omega_{s} \rightarrow \Omega_{r}$, being $s, r \in I$, as

$$
\mathscr{A}_{r}^{s}=\mathscr{A}_{r} \circ \mathscr{A}_{s}^{-1} \text {. }
$$

For the sake of notation, however, whenever we have to integrate a quantity $u(r): \Omega_{r} \rightarrow \mathbb{R}$ on a configuration $\Omega_{s}$ with $s \neq r$, we will simply write $\int_{\Omega_{s}} u(r) \mathrm{d} \Omega$ instead of the (formally more correct) expression $\int_{\Omega_{s}} u(r) \circ \mathscr{A}_{r}^{s} \mathrm{~d} \Omega$. Analogously, we will use $\|u(r)\|_{L^{2}\left(\Omega_{s}\right)}$ as a short-hand notation of $\left\|u(r) \circ \mathscr{A}_{r}^{s}\right\|_{L^{2}\left(\Omega_{s}\right)}$ and so on.

\subsection{Finite element ALE formulation}

We briefly recall the set up for the finite element formulation in an ALE frame that has been developed in [4]. 
Let $\mathscr{X}_{h}\left(\Omega_{0}\right) \subset H^{1}\left(\Omega_{0}\right)$ be a finite element space defined on the reference configuration $\Omega_{0}$. The finite element space on the current configuration is constructed by the help of the ALE mapping as

$$
\mathscr{X}_{h}\left(\Omega_{t}\right)=\left\{\psi_{h}: \Omega_{t} \times I \rightarrow \mathbb{R}, \quad \psi_{h}=\widehat{\psi}_{h} \circ \mathscr{A}_{t}^{-1}, \quad \widehat{\psi}_{h} \in \mathscr{X}_{h}\left(\Omega_{0}\right)\right\} .
$$

In most cases, the ALE mapping is, on its turn, represented by means of finite elements and is computed, for instance, by solving a suitable PDE, given the displacement of the domain boundary. Clearly, if this is the case, the domain boundary will not be exactly represented. Yet since this aspect is not crucial for the analysis that we will carry out in this paper, we will continue to indicate the computational domain by $\Omega_{t}$, for the sake of simplicity.

Let $\mathscr{N}$ be the set of nodes of the finite element mesh and $\mathscr{N}_{\text {int }} \subset \mathscr{N}$ that containing just the internal nodes. The set of Lagrange basis functions on the reference configuration $\Omega_{0}$ is indicated by

$$
\left\{\hat{\psi}_{i}, \hat{\psi}_{i} \in \mathscr{X}_{h}\left(\Omega_{0}\right), i \in \mathscr{N}\right\},
$$

and forms a basis of $\mathscr{X}_{h}\left(\Omega_{0}\right)$, while $\left\{\hat{\psi}_{i}, i \in \mathscr{N}_{\text {int }}\right\}$ forms a basis of $\mathscr{X}_{0, h}\left(\Omega_{0}\right)=\mathscr{X}_{h}\left(\Omega_{0}\right) \cap H_{0}^{1}\left(\Omega_{0}\right)$.

Correspondingly, we indicate with $\psi_{i}$ the finite element basis function on the triangulation of $\Omega_{t}$, defined as

$$
\psi_{i}=\hat{\psi}_{i} \circ \mathscr{A}_{t}^{-1}, \quad i \in \mathscr{N} .
$$

For each $t \in I$ the numerical solution $u_{h}$ may then be expressed as a linear combination of nodal finite element basis functions, i.e.

$$
u_{h}(\mathbf{x}, t)=\sum_{i \in \mathscr{N}} \psi_{i}(\mathbf{x}, t) u_{i}(t)
$$

with time dependent coefficients $u_{i}(t)$. The major difference with standard finite element formulation for time-dependent problems is that here the finite element basis functions depend on time because of the ALE mapping.

Then, the finite element approximation of the non-conservative formulation (6) may be written as

\section{Semi-discrete non-conservative formulation}

For all $t \in I$, find $u_{h} \in \mathscr{X}_{h}\left(\Omega_{t}\right)$ such that

$$
\left.\int_{\Omega_{t}} \frac{\partial u_{h}}{\partial t}\right|_{\mathbf{Y}} \psi_{h} \mathrm{~d} \Omega+\mu \int_{\Omega_{t}} \nabla_{\mathbf{x}} u_{h} \cdot \nabla_{\mathbf{x}} \psi_{h} \mathrm{~d} \Omega+\int_{\Omega_{t}}\left[\nabla_{\mathbf{x}} \cdot\left(\boldsymbol{\beta} u_{h}\right)-\mathbf{w} \cdot \nabla_{\mathbf{x}} u_{h}\right] \psi_{h} \mathrm{~d} \Omega=\int_{\Omega_{t}} f \psi_{h} \mathrm{~d} \Omega \quad \forall \psi_{h} \in \mathscr{X}_{0, h}\left(\Omega_{t}\right)
$$

with

$$
\begin{aligned}
& u_{h}=u_{\mathrm{D} h} \quad \text { for } \mathbf{x} \in \partial \Omega_{t}, \quad t \in I \\
& u_{h}=u_{0 h} \quad \text { for } \mathbf{x} \in \Omega_{0}, \quad t=t_{0} .
\end{aligned}
$$

$u_{\mathrm{D}, h}$ and $u_{0, h}$ being suitable finite element approximations of $u_{\mathrm{D}}$ and $u_{0}$, respectively.

By denoting by $U=\left\{u_{i}\right\}_{i \in \mathcal{N}}$ the vector of nodal values of $u_{h}$ (which are, of course, functions of time), we may express (11) in an algebraic form as

$$
\mathbf{M}(t) \frac{\mathrm{d} \mathbf{U}(t)}{\mathrm{d} t}+\mathbf{H}(t) \mathbf{U}(t)-\mathbf{B}(t, \mathbf{w}(t)) \mathbf{U}(t)=\mathbf{F}(t), \quad t \in I
$$

plus

$$
u_{i}(t)=u_{\mathrm{D}, h}\left(\mathbf{x}_{i}, t\right), \quad i \in \mathscr{N} \backslash \mathscr{N}_{\mathrm{int}}
$$


and

$$
u_{i}\left(t_{0}\right)=u_{0, h}\left(\mathbf{x}_{i}\right), \quad i \in \mathscr{N} .
$$

Here,

$$
\mathbf{M}(t)=\left\{\int_{\Omega_{t}} \psi_{i}(t) \psi_{j}(t) \mathrm{d} \Omega\right\}_{i \in \mathcal{N}_{\text {int }}, j \in \mathcal{N}}
$$

is the mass matrix, while $\mathbf{H}$ and $\mathbf{B}$ are defined as

$$
\begin{aligned}
& \mathbf{H}(t)=\left\{\int_{\Omega_{t}} \nabla_{\mathbf{x}} \cdot\left(\boldsymbol{\beta} \psi_{j}(t)\right) \psi_{i}(t) \mathrm{d} \Omega+\mu \int_{\Omega_{t}} \nabla_{\mathbf{x}} \psi_{j}(t) \cdot \nabla_{\mathbf{x}} \psi_{i}(t) \mathrm{d} \Omega\right\}_{i \in \mathcal{N}_{\text {int }, j \in \mathcal{N}}}, \\
& \mathbf{B}(t, \mathbf{w})=\left\{\int_{\Omega_{t}}\left(\mathbf{w}(t) \cdot \nabla_{\mathbf{x}} \psi_{j}(t)\right) \psi_{i}(t) \mathrm{d} \Omega\right\}_{i \in \mathcal{N}_{\text {int }}, j \in \mathcal{N}},
\end{aligned}
$$

and

$$
\mathbf{F}(t)=\left\{\int_{\Omega_{t}} f(t) \psi_{i}(t) \mathrm{d} \Omega\right\}_{i \in \mathcal{N}_{\text {int }}} .
$$

This formulation differs from the one usually obtained in the finite element method when the unknowns on the Dirichlet portion of the boundary are statically eliminated, producing a system with square matrices $\mathbf{M}, \mathbf{H}$ and $\mathbf{B}$ and a modified right-hand side. Yet, this equivalent formulation is here preferred for the further discussion.

On the other hand, the finite element approximation of the conservative formulation (8) may be written as

\section{Semi-discrete conservative formulation}

For each $t \in I$, find $u_{h} \in \mathscr{X}_{h}\left(\Omega_{t}\right)$ such that

$$
\frac{\mathrm{d}}{\mathrm{d} t} \int_{\Omega_{t}} u_{h} \psi_{h} \mathrm{~d} \Omega+\mu \int_{\Omega_{t}} \nabla_{\mathbf{x}} u_{h} \cdot \nabla_{\mathbf{x}} \psi_{h} \mathrm{~d} \Omega+\int_{\Omega_{t}} \nabla_{\mathbf{x}} \cdot\left[(\boldsymbol{\beta}-\mathbf{w}) u_{h}\right] \psi_{h} \mathrm{~d} \Omega=\int_{\Omega_{t}} f \psi_{h} \mathrm{~d} \Omega \forall \psi_{h} \in \mathscr{X}_{0, h}\left(\Omega_{t}\right),
$$

with (11a) and (11b) as initial and boundary conditions, respectively.

Equivalently, the algebraic form reads:

$$
\frac{\mathrm{d}}{\mathrm{d} t}(\mathbf{M}(t) \mathbf{U}(t))+(\mathbf{H}(t)-\mathbf{A}(t, \mathbf{w}(t))-\mathbf{B}(t, \mathbf{w}(t))) \mathbf{U}(t)=\mathbf{F}(t) .
$$

Here,

$$
\mathbf{A}(t, \mathbf{w})=\left\{\int_{\Omega_{t}} \nabla_{\mathbf{x}} \cdot \mathbf{w}(t) \psi_{j}(t) \psi_{i}(t) \mathrm{d} \Omega\right\}_{i \in \mathcal{N}_{\text {int }}, j \in \mathcal{N}} .
$$

Both (12) and (15) are systems of ordinary differential equations and they may be put into the general form

$$
\frac{\mathrm{d} \mathbf{y}(t)}{\mathrm{d} t}=\mathbf{g}_{1}(t, \mathbf{U}(t))+\mathbf{g}_{\mathbf{w}}(t, \mathbf{U}(t)), \quad t \in I,
$$

where $\mathbf{g}_{1}(t, \mathbf{U}(t))$ accounts for the diffusive and convective terms, $\mathbf{g}_{\mathbf{w}}(t, \mathbf{U}(t))$ is the term that depends on the domain velocity $\mathbf{w}$, while 


$$
\mathbf{y}(t)= \begin{cases}\mathbf{U}(t) & \text { non-conservative formulation } \\ \mathbf{M}(t) \mathbf{U}(t), & \text { conservative formulation }\end{cases}
$$

We observe that the differential system is not autonomous even in the case of steady boundary conditions and forcing term, since $\mathbf{g}_{1}$ and $\mathbf{g}_{\mathbf{w}}$ depend on time not only through $\mathbf{U}$, but also because of the domain movement.

We now recall the definition of the Geometric Conservation Laws (GCL) as given, for instance in $[4,8,13]$.

Definition 1. A numerical scheme posed on a moving domain satisfies the Geometric Conservation Laws if it is able to reproduce exactly a constant solution.

Clearly, we are assuming that the constant solution is admissible for the problem at hand. This is indeed the case whenever $f=0$ and the boundary and initial conditions are appropriately chosen. It has been shown in [4] that the non-conservative formulation (12) satisfies the GCL provided that all space integrals are computed exactly, irrespectively to the time-advancing scheme adopted.

On the contrary, the conservative formulation (15) satisfies the GCL only if the identity

$$
\frac{\mathrm{d}}{\mathrm{d} t} \int_{\Omega_{t}} \psi_{i} \mathrm{~d} \Omega=\int_{\Omega_{t}} \psi_{i} \nabla_{\mathbf{x}} \cdot \mathbf{w} \mathrm{d} \Omega, \quad \forall i \in \mathscr{N}_{\text {int }}
$$

which expresses the conservation of purely geometrical quantities, is satisfied at discrete level. Algebraically (17) is equivalent to

$$
\frac{\mathrm{d}}{\mathrm{d} t}(\mathbf{M}(t) \mathbf{1})=\mathbf{g}_{\mathbf{w}}(t, \mathbf{1})=\mathbf{A}(t, \mathbf{w}(t)) \mathbf{1}
$$

where $\mathbf{1} \in \mathbb{R}^{N}$ indicates a vector of all unit elements, being $N=\operatorname{card}(\mathscr{N})$. We may then affirm that a time advancing scheme satisfies the GCL whenever it solves exactly the problem

$$
\left\{\begin{array}{l}
\frac{\mathrm{d} \mathbf{M}(t) \mathbf{z}(t)}{\mathrm{d} t}=\mathbf{A}(t, \mathbf{w}(t)) \mathbf{z}(t), \quad t \in I \\
\mathbf{z}\left(t_{0}\right)=\mathbf{1}
\end{array}\right.
$$

i.e. it should return $\mathbf{z}^{n}=\mathbf{z}\left(t^{n}\right)=\mathbf{1}$ for all $n$ such that $t^{n} \leqslant T$.

In [4] we proposed another, stronger, characterization of the GCL that is more appropriate in a finite element context. Precisely, it is required that the discrete system should satisfy exactly the identity

$$
\frac{\mathrm{d}}{\mathrm{d} t} \int_{\Omega_{t}} \psi_{i} \psi_{j} \mathrm{~d} \Omega=\int_{\Omega_{t}} \psi_{i} \psi_{j} \nabla_{\mathbf{x}} \cdot \mathbf{w} \mathrm{d} \Omega, \quad \forall i, j \in \mathscr{N} .
$$

In particular, this implies that, for any vector $\mathbf{d} \in \mathbb{R}^{N}$, the time advancing scheme should be able to solve exactly

$$
\left\{\begin{array}{l}
\frac{\mathrm{d} \mathbf{M}(t) \mathbf{z}(t)}{\mathrm{d} t}=\mathbf{A}(t, \mathbf{w}(t)) \mathbf{z}(t), \quad t \in I \\
\mathbf{z}\left(t_{0}\right)=\mathbf{d},
\end{array}\right.
$$

i.e. it should return $\mathbf{z}^{n}=\mathbf{z}\left(t^{n}\right)=\mathbf{d}$ for all $n$ such that $t^{n} \leqslant T$. 


\section{First-order time discretization schemes}

Let $t_{0}<t_{1}<\cdots<t_{M}=T$ be a uniform partition of $I=\left[t_{0}, T\right]$ with time step $\Delta t$ and $\mathbf{y}^{n}$ an approximation of $\mathbf{y}\left(t^{n}\right)$. In [4] we have analyzed the following modified implicit Euler scheme,

$$
\mathbf{y}^{n+1}-\mathbf{y}^{n}=\Delta t \sum_{l=0}^{m} \omega_{l}\left[\mathbf{g}_{1}\left(t_{l}^{n}, \mathbf{U}^{n+1}\right)+\mathbf{g}_{\mathbf{w}}\left(t_{l}^{n}, \mathbf{U}^{n+1}\right)\right],
$$

where $\omega_{l}$ and $t_{l}^{n}$ are the weights and knots of a quadrature rule $\mathscr{I}$ in $\left[t^{n}, t^{n+1}\right]$ such that

$$
\mathscr{I}(f)=\Delta t \sum_{l=0}^{m} \omega_{l} f\left(t_{l}^{n}\right) \approx \int_{t^{n}}^{t^{n+1}} f(\tau) \mathrm{d} \tau .
$$

Observe that, if the quadrature rule is taken simply as $\mathscr{I}(f)=\Delta t f\left(t^{n+1}\right)$, we recover the classic implicit Euler scheme.

In the case of the conservative formulation $(\mathbf{y}=\mathbf{M U})$, it is immediately verified that scheme (21), when applied to (18), satisfies the GCL if and only if the chosen quadrature rule integrates exactly the term $\int_{t^{n}}^{t^{n+1}} \mathbf{A}(s, \mathbf{w}(s)) \mathrm{d} s$, that is, if the following relation holds $\forall i \in \mathscr{N}_{\text {int }}$

$$
\Delta t \sum_{l=0}^{m} \omega_{l} \int_{\Omega_{t_{l}^{n}}} \psi_{i}\left(t_{l}^{n}\right) \boldsymbol{\nabla}_{\mathbf{x}} \cdot \mathbf{w}\left(t_{l}^{n}\right) \mathrm{d} \Omega=\int_{t^{n}}^{t^{n+1}} \int_{\Omega_{s}} \psi_{i}(s) \nabla_{\mathbf{x}} \cdot \mathbf{w}(s) \mathrm{d} \Omega \mathrm{d} s .
$$

This relation guarantees that the conservation of the geometric quantities expressed by (17) also holds at discrete level.

It can be shown [4] that (22) also implies, for all $i, j \in \mathscr{N}$,

$$
\Delta t \sum_{l=0}^{m} \omega_{l} \int_{\Omega_{t_{l}^{n}}} \psi_{i}\left(t_{l}^{n}\right) \psi_{j}\left(t_{l}^{n}\right) \boldsymbol{\nabla}_{\mathbf{x}} \cdot \mathbf{w}\left(t_{l}^{n}\right) \mathrm{d} \Omega=\int_{t^{n}}^{t^{n+1}} \int_{\Omega_{s}} \psi_{i}(s) \psi_{j}(s) \boldsymbol{\nabla}_{\mathbf{x}} \cdot \mathbf{w}(s) \mathrm{d} \Omega \mathrm{d} s,
$$

and consequently the time advancing scheme would solve exactly (20) as well.

Apart from very particular cases, the domain movement, and thus $\mathscr{A}_{t}$ and $\mathbf{w}$, is not known a priori. For instance, this is the situation in fluid structure interaction or free surface flow problems. In these cases, however, in the course of the computation, the position of the computational domain boundary is given at time stations $t^{j}$, with $j=0,1, \ldots, M$, and an ALE mapping may be computed at those instants, for instance by employing an harmonic extension of the boundary position.

The mapping $\mathscr{A}_{s}$ at intermediate instants $s \in\left[t^{j}, t^{j+1}\right]$ may then be obtained by interpolation from $\mathscr{A}_{t^{j+1}}, \mathscr{A}_{t^{j}}, \mathscr{A}_{t^{j-1}}, \ldots$ with a polynomial of degree $p \geqslant 1$. Observe that the reconstructed ALE mapping $\mathscr{A}_{s}, \forall s \in\left[t^{j}, t^{j+1}\right]$ provides automatically the reconstruction for the domain velocity since $\mathbf{w}=\left.\frac{\partial \mathscr{A}_{s}}{\partial s}\right|_{\mathbf{Y}}$. It is then reasonable to assume that the ALE map, and the domain velocity, is a piecewise polynomial in time.

The following two results have been given in [4]:

Proposition 1. A sufficient condition for the scheme (21) to fulfill the GCL (i.e. to satisfy (22), or equivalently, (23)) is to use a quadrature rule $\mathscr{I}$ of degree of exactness equal or grater than $d \cdot p-1$, where $d$ is the space dimension and $p$ is the degree of the polynomial used to reconstruct in time the ALE mapping within each time step.

For a linear in time reconstruction of the ALE mapping $(p=1)$ a mid-point quadrature rule is sufficient for a 2D problem to fulfill the GCL, whereas in a 3D problem we should employ, for instance, a two point Gaussian quadrature rule. 
Proposition 2. Whenever the scheme (21) is applied to the conservative formulation (15) and the quadrature rule $\mathscr{I}$ is sufficient to fulfill the $G C L$ and has positive weights $\omega_{l}, l=0, \ldots, m$, then the scheme (21) is unconditionally stable and the following a priory estimate holds:

$$
\left\|u_{h}^{n+1}\right\|_{L_{2}\left(\Omega_{t^{n+1}}\right)}^{2}+\Delta t \mu \sum_{i=0}^{n} \sum_{l=0}^{m} \omega_{l}\left\|\nabla_{\mathbf{x}} u_{h}^{i+1}\right\|_{L_{2}\left(\Omega_{i_{l}}\right)}^{2} \leqslant\left\|u_{h}^{0}\right\|_{L_{2}\left(\Omega_{t^{0}}\right)}^{2}+\Delta t \frac{\left(1+C_{\Omega}\right)}{\mu} \sum_{i=0}^{n} \sum_{l=0}^{m} \omega_{l}\left\|f\left(t_{l}^{i}\right)\right\|_{H^{-1}\left(\Omega_{i_{l}}{ }^{i}\right.}^{2} .
$$

Remark 2. The quantity $u_{h}^{n}=\sum_{i \in \mathcal{N}} u_{i}^{n} \psi_{i}\left(t^{n}\right)$ may be readily extended to any other configuration $\Omega(s)$ with $s \neq t^{n}$, since

$$
u_{h}^{n} \circ \mathscr{A}_{t^{n}}^{s}=\sum_{i \in \mathcal{N}} u_{i}^{n} \psi_{i}(s)
$$

This extension has been implicitly assumed whenever required.

In the cited reference it is also shown that the same scheme (21), applied to the non-conservative formulation (12) is, in general, only conditionally stable, with a maximum allowable time step that depends on the domain velocity $\mathbf{w}$.

Remark 3. The results of Proposition 2 also holds if the quadrature rule with the appropriate degree of exactness is applied just to the term $\mathbf{g}_{\mathbf{w}}$, which is the only one actually involved in the GCL. In particular, we could have considered the following alternative GCL-satisfying scheme

$$
\mathbf{y}^{n+1}=\mathbf{y}^{n}+\Delta t \mathbf{g}_{1}\left(t^{n+1}, \mathbf{U}^{n+1}\right)+\Delta t \sum_{l=0}^{m} \omega_{l} \mathbf{g}_{\mathbf{w}}\left(t_{l}^{n}, \mathbf{U}^{n+1}\right) .
$$

It can be easily shown that an a priori stability inequality very similar to (24) can be obtained as well. Yet, scheme (21) may be more convenient from a programming point of view since we will treat all the terms in the same way.

\section{Second-order time discretization schemes}

The stability result given in the previous section shows that for the implicit Euler scheme the GCL are sufficient conditions to preserve the stability properties that the scheme features when applied to a parabolic problem on a fixed domain. Unfortunately, we will see in this section that this remarkable result is not so general as we would like. We will here consider two second-order time discretization schemes, namely, the Crank-Nicolson and the second-order backward difference (hereafter BDF(2)). Both of them are unconditionally stable when applied to problems on a fixed domain. We will show that, even in the case where the GCL are satisfied, standard techniques to produce energy estimates of the numerical solution lead to inequalities where a few terms, deriving from the domain movement, have a sign that cannot be a priori determined. The numerical tests presented in a later section confirm that an additional energy contribution to the numerical solution may occur because of the boundary movement. We point out that in the a priori estimate (2) the domain deformation does not contribute to the energy of the system in the differential problem, therefore this extra energy contribution is a numerical artifact. 


\subsection{The Crank-Nicolson method}

We will consider here a slight modification to the classical Crank-Nicolson method that, for the problem (16) reads:

$$
\mathbf{y}^{n+1}-\mathbf{y}^{n}=\Delta t\left[\mathbf{g}_{1}\left(t^{n+\frac{1}{2}}, \frac{\mathbf{U}^{n+1}+\mathbf{U}^{n}}{2}\right)+\mathbf{g}_{\mathbf{w}}\left(t^{n+\frac{1}{2}}, \frac{\mathbf{U}^{n+1}+\mathbf{U}^{n}}{2}\right)\right] .
$$

More precisely, this scheme is a Gauss-Legendre implicit Runge-Kutta method of order 2. However, with a little abuse on notations, we will refer to it as the Crank-Nicolson method, since it reduces to the latter for a linear advection-diffusion equation on a fixed domain and with time-independent coefficients.

Observe that the right hand side of (26) is an approximation of

$$
\int_{t^{n}}^{t^{n+1}}\left[\mathbf{g}_{1}(\tau, \mathbf{U}(\tau))+\mathbf{g}_{\mathbf{w}}(\tau, \mathbf{U}(\tau))\right] \mathrm{d} \tau
$$

by means of a mid-point rule. For 2D problems, this approximation, when applied to the conservative formulation, will automatically satisfy the GCL if the ALE mapping is reconstructed linearly in time in each time slab.

In the more general case, we should employ a more accurate quadrature rule, at least for the terms in $\mathbf{g}_{\mathbf{w}}$ that are related to the domain movement, according to the criterion given in Proposition 1. For instance we may use

$$
\mathbf{y}^{n+1}-\mathbf{y}^{n}=\Delta t \sum_{l=0}^{m} \omega_{l}\left[\mathbf{g}_{1}\left(t_{l}^{n}, \frac{\mathbf{U}^{n+1}+\mathbf{U}^{n}}{2}\right)+\mathbf{g}_{\mathbf{w}}\left(t_{l}^{n}, \frac{\mathbf{U}^{n+1}+\mathbf{U}^{n}}{2}\right)\right] .
$$

Here $m, t_{l}^{n}$ and $\omega_{l}$ are chosen so that the resulting scheme satisfies the GCL.

We are going now to study the stability of this scheme, when applied to the conservative formulation with homogeneous boundary conditions. Scheme (27) is then equivalent to

$$
\begin{aligned}
& \int_{\Omega_{t^{n+1}}} u_{h}^{n+1} \psi_{h} \mathrm{~d} \Omega-\int_{\Omega_{t^{n}}} u_{h}^{n} \psi_{h} \mathrm{~d} \Omega+\Delta t \sum_{l=0}^{m} \omega_{l} \int_{\Omega_{t_{l}^{n}}} \mu \nabla_{\mathbf{x}} \frac{u_{h}^{n+1}+u_{h}^{n}}{2} \cdot \nabla_{\mathbf{x}} \psi_{h} \mathrm{~d} \Omega \\
& +\Delta t \sum_{l=0}^{m} \omega_{l} \int_{\Omega_{t_{l}^{n}}} \nabla_{\mathbf{x}} \cdot\left[\left(\boldsymbol{\beta}-\mathbf{w}\left(t_{l}^{n}\right)\right) \frac{u_{h}^{n+1}+u_{h}^{n}}{2}\right] \psi_{h} \mathrm{~d} \Omega \\
& =\Delta t \sum_{l=0}^{m} \omega_{l} \int_{\Omega_{t_{l}^{n}}} f\left(t_{l}^{n}\right) \psi_{h} \mathrm{~d} \Omega, \quad \forall \psi_{h} \in \mathscr{X}_{0, h}\left(\Omega_{t}\right), \quad n=0,1, \ldots
\end{aligned}
$$

with

$$
\begin{aligned}
& u_{h}^{i}=0 \quad \text { on } \partial \Omega_{t}, \quad i \in \mathscr{N} \backslash \mathscr{N}_{\mathrm{int}} \\
& u_{h}^{0}=u_{0 h} \quad \text { in } \Omega_{0} .
\end{aligned}
$$

As mentioned before, this scheme satisfies the GCL. Thus, an immediate consequence of (23) is that

$$
\left\|v_{h}\right\|_{L_{2}\left(\Omega_{t^{n+1}}\right)}^{2}-\left\|v_{h}\right\|_{L_{2}\left(\Omega_{t^{n}}\right)}^{2}=\Delta t \sum_{l=0}^{m} \omega_{l} \int_{\Omega_{t_{l}^{n}}}\left|v_{h}\right|^{2} \nabla_{\mathbf{x}} \cdot \mathbf{w}\left(t_{l}^{n}\right) \mathrm{d} \Omega .
$$

for any finite element function $v_{h}(\mathbf{x}, t)=\sum_{i \in \mathcal{N}} v_{i} \psi_{i}(\mathbf{x}, t)$ with coefficients $v_{i}$ that do not depend on $t$. 
We will first derive the following result:

Lemma 1. The discrete solution $u_{h}^{n}$ of scheme (28) satisfies the inequality

$$
\begin{aligned}
& \left\|u_{h}^{n+1}\right\|_{L_{2}\left(\Omega_{t^{n+1}}\right)}^{2}+\frac{\Delta t}{4} \mu \sum_{l=0}^{m} \omega_{l}\left\|\nabla_{\mathbf{x}}\left(u_{h}^{n+1}+u_{h}^{n}\right)\right\|_{L_{2}\left(\Omega_{l_{l}^{n}}\right)}^{2}-\frac{\Delta t}{4} \sum_{l=0}^{m} \omega_{l} \int_{\Omega_{l_{l}^{n}}} \nabla_{\mathbf{x}} \cdot \mathbf{w}\left(t_{l}^{n}\right)\left|u_{h}^{n+1}-u_{h}^{n}\right|^{2} \mathrm{~d} \Omega \\
& \quad \leqslant\left\|u_{h}^{n}\right\|_{L_{2}\left(\Omega_{t^{n}}\right)}^{2}+\Delta t \frac{\left(1+C_{\Omega}\right)}{\mu} \sum_{l=0}^{m} \omega_{l}\left\|f\left(t_{l}^{n}\right)\right\|_{H^{-1}\left(\Omega_{t_{l}^{n}}\right)}^{2}
\end{aligned}
$$

for all $n=0,1, \ldots$

Proof. We take in (28) $\psi_{h}=\left(u_{h}^{n+1}+u_{h}^{n}\right)$ and we use the identity

$$
(a, a+b)=\frac{1}{2}\|a\|^{2}+\frac{1}{2}\|a+b\|^{2}-\frac{1}{2}\|b\|^{2}
$$

to write the first two terms in (28) as

$$
\begin{aligned}
\int_{\Omega_{t^{n+1}}} & u_{h}^{n+1}\left(u_{h}^{n+1}+u_{h}^{n}\right) \mathrm{d} \Omega-\int_{\Omega_{t^{n}}} u_{h}^{n}\left(u_{h}^{n+1}+u_{h}^{n}\right) \mathrm{d} \Omega \\
= & \frac{1}{2}\left\|u_{h}^{n+1}\right\|_{L_{2}\left(\Omega_{t^{n+1}}\right)}^{2}+\frac{1}{2}\left\|u_{h}^{n+1}+u_{h}^{n}\right\|_{L_{2}\left(\Omega_{t^{n+1}}\right)}^{2}-\frac{1}{2}\left\|u_{h}^{n}\right\|_{L_{2}\left(\Omega_{t^{n+1}}\right)}^{2}-\frac{1}{2}\left\|u_{h}^{n}\right\|_{L_{2}\left(\Omega_{t^{n}}\right)}^{2} \\
& -\frac{1}{2}\left\|u_{h}^{n+1}+u_{h}^{n}\right\|_{L_{2}\left(\Omega_{t^{n}}\right)}^{2}+\frac{1}{2}\left\|u_{h}^{n+1}\right\|_{L_{2}\left(\Omega_{t^{n}}\right)}^{2} \\
= & \left\|u_{h}^{n+1}\right\|_{L_{2}\left(\Omega_{t^{n+1}}\right)}^{2}-\left\|u_{h}^{n}\right\|_{L_{2}\left(\Omega_{t^{n}}\right)}^{2}-\frac{1}{2} \Delta t \sum_{l=0}^{m} \omega_{l} \int_{\Omega_{t_{l}^{n}}} \nabla_{\mathbf{x}} \cdot \mathbf{w}\left(t_{l}^{n}\right)\left|u_{h}^{n+1}\right|^{2} \mathrm{~d} \Omega \\
& -\frac{1}{2} \Delta t \sum_{l=0}^{m} \omega_{l}\left[\int_{\Omega_{t_{l}^{n}}} \nabla_{\mathbf{x}} \cdot \mathbf{w}\left(t_{l}^{n}\right)\left|u_{h}^{n}\right|^{2} \mathrm{~d} \Omega-\int_{\Omega_{t_{l}^{n}}} \nabla_{\mathbf{x}} \cdot \mathbf{w}\left(t_{l}^{n}\right)\left|u_{h}^{n+1}+u_{h}^{n}\right|^{2} \mathrm{~d} \Omega\right] \\
= & \left\|u_{h}^{n+1}\right\|_{L_{2}\left(\Omega_{t^{n+1}}\right)}^{2}-\left\|u_{h}^{n}\right\|_{L_{2}\left(\Omega_{t^{n}}\right)}^{2}+\Delta t \sum_{l=0}^{m} \omega_{l} \int_{\Omega_{t_{l}^{n}}} \nabla_{\mathbf{x}} \cdot \mathbf{w}\left(t_{l}^{n}\right) u_{h}^{n+1} u_{h}^{n} \mathrm{~d} \Omega,
\end{aligned}
$$

where we have exploited relation (29) with $v_{h}=u_{h}^{n+1}, v_{h}=u_{h}^{n}$, and $v_{h}=\left(u_{h}^{n+1}+u_{h}^{n}\right)$. Then, Eq. (28), with $\psi_{h}=\left(u_{h}^{n+1}+u_{h}^{n}\right)$, becomes, after integration by parts of the convective term

$$
\begin{aligned}
& \left\|u_{h}^{n+1}\right\|_{L_{2}\left(\Omega_{t^{n+1}}\right)}^{2}-\left\|u_{h}^{n}\right\|_{L_{2}\left(\Omega_{t^{n}}\right)}^{2}+\Delta t \sum_{l=0}^{m} \omega_{l} \int_{\Omega_{t_{l}^{n}}} \nabla_{\mathbf{x}} \cdot \mathbf{w}\left(t_{l}^{n}\right) u_{h}^{n+1} u_{h}^{n} \mathrm{~d} \Omega \\
& +\Delta t \frac{\mu}{2} \sum_{l=0}^{m} \omega_{l}\left\|\nabla_{\mathbf{x}}\left(u_{h}^{n+1}+u_{h}^{n}\right)\right\|_{L_{2}\left(\Omega_{t_{l}^{n}}\right)}^{2}-\frac{\Delta t}{4} \sum_{l=0}^{m} \omega_{l} \int_{\Omega_{t_{l}^{n}}} \nabla_{\mathbf{x}} \cdot \mathbf{w}\left(t_{l}^{n}\right)\left|u_{h}^{n+1}+u_{h}^{n}\right|^{2} \mathrm{~d} \Omega \\
& \leqslant \Delta t \frac{\left(1+C_{\Omega}\right)}{\mu} \sum_{l=0}^{m} \omega_{l}\left\|f\left(t_{l}^{n}\right)\right\|_{H^{-1}\left(\Omega_{t_{l}^{n}}\right)}^{2}+\Delta t \frac{\mu}{4} \sum_{l=0}^{m} \omega_{l}\left\|\nabla_{\mathbf{x}}\left(u_{h}^{n+1}+u_{h}^{n}\right)\right\|_{L_{2}\left(\Omega_{t_{l}^{n}}\right)}^{2}
\end{aligned}
$$

and the thesis follows immediately.

Remark 4. This modified version of the Crank-Nicolson scheme allowed us to exploit directly the coercivity of the bilinear form stemming from the Laplace operator to obtain our result. This would have not been possible with the standard scheme, as a consequence of the domain movement. 
Remark 5. If $\nabla_{\mathbf{x}} \cdot \mathbf{w}<0, \forall \mathbf{x} \in \Omega_{t}$ and $\forall t \in I$ (i.e. in the case of a uniform contraction of the domain), we can obtain from (30) an unconditioned stability result like the one previously obtained for the implicit Euler scheme. In the more general case we can only obtain a conditioned stability where the maximum allowable time step depends on $\boldsymbol{\nabla}_{\mathbf{x}} \cdot \mathbf{w}$. More details may be found in [10].

Remark 6. In the previous example we enforced the GCL by employing a more accurate quadrature rule to both the terms $\mathbf{g}_{1}$ and $\mathbf{g}_{\mathbf{w}}$. Clearly, we could have applied the more sophisticated quadrature rule only to the term $\mathbf{g}_{\mathbf{w}}$. This is enough, indeed, to fulfill the GCL. Yet, the former scheme may be preferable from a programming point of view since we will treat all terms in the same way.

\subsection{The second-order backward difference scheme BDF(2)}

The second-order backward difference scheme applied to (16) reads:

$$
\frac{3}{2} \mathbf{y}^{n+1}-2 \mathbf{y}^{n}+\frac{1}{2} \mathbf{y}^{n-1}=\mathbf{g}_{1}\left(t^{n+1}, \mathbf{U}^{n+1}\right)+\mathbf{g}_{\mathbf{w}}\left(t^{n+1}, \mathbf{U}^{n+1}\right), \quad n \geqslant 1 .
$$

This scheme can be initialized by a one-step second-order method such as, for instance, the CrankNicolson one presented in the previous section.

This scheme does not satisfy the GCL in general, when applied to the conservative formulation. However, we observe that

$$
\frac{3}{2} \mathbf{y}^{n+1}-2 \mathbf{y}^{n}+\frac{1}{2} \mathbf{y}^{n-1}=\frac{3}{2} \int_{t^{n}}^{t^{n+1}} \mathbf{g}(\tau, \mathbf{U}(\tau)) \mathrm{d} \tau-\frac{1}{2} \int_{t^{n-1}}^{t^{n}} \mathbf{g}(\tau, \mathbf{U}(\tau)) \mathrm{d} \tau,
$$

where we have set $\mathbf{g}=\mathbf{g}_{1}+\mathbf{g}_{\mathbf{w}}$. We can thus modify scheme (33) by using a more accurate quadrature rule to integrate the two terms in the right hand side (while keeping $\mathbf{U}(t)=\mathbf{U}^{n+1}$ ). If we carry out this procedure on the whole $\mathbf{g}$, however, we will destroy the coerciveness of the form $\mathbf{g}_{1}$ since the second integral appears with a negative sign. Consequently, to impose the GCL compliance to the BDF(2) scheme we are obliged to operate on the term $\mathbf{g}_{\mathbf{w}}$ only. The modified scheme reads then, in a general form:

$$
\frac{3}{2} \mathbf{y}^{n+1}-2 \mathbf{y}^{n}+\frac{1}{2} \mathbf{y}^{n-1}=\mathbf{g}_{1}\left(t^{n+1}, \mathbf{U}^{n+1}\right)+\frac{3}{2} \Delta t \sum_{l=0}^{m} \omega_{l} \mathbf{g}_{\mathbf{w}}\left(t_{l}^{n}, \mathbf{U}^{n+1}\right)-\frac{1}{2} \Delta t \sum_{l=0}^{m} \omega_{l} \mathbf{g}_{\mathbf{w}}\left(t_{l}^{n-1}, \mathbf{U}^{n+1}\right) .
$$

This scheme satisfies the GCL provided that the quadrature rule employed for $\mathbf{g}_{\mathbf{w}}$ fulfills the requirements given in Proposition 1.

This scheme has already been proposed by Farhat and Koobus [7] in the context of a finite volume approximation.

In the remaining part of this section, we will derive a stability result for the BDF(2) scheme (35).

Again, for the sake of clarity, we rewrite this scheme in the equivalent form

$$
\begin{aligned}
& \frac{3}{2} \int_{\Omega_{t^{n+1}}} u_{h}^{n+1} \psi_{h} \mathrm{~d} \Omega-2 \int_{\Omega_{t^{n}}} u_{h}^{n} \psi_{h} \mathrm{~d} \Omega+\frac{1}{2} \int_{\Omega_{t^{n-1}}} u_{h}^{n-1} \psi_{h} \mathrm{~d} \Omega \\
& \quad+\Delta t \int_{\Omega_{t^{n+1}}}\left[\mu \nabla_{\mathbf{x}} u_{h}^{n+1} \cdot \nabla_{\mathbf{x}} \psi_{h}+\nabla_{\mathbf{x}} \cdot\left(\boldsymbol{\beta} u_{h}^{n+1}\right)\right] \psi_{h} \mathrm{~d} \Omega \\
& \quad-\frac{3}{2} \Delta t \sum_{l=0}^{m} \omega_{l} \int_{\Omega_{t_{l}^{n}}} \nabla_{\mathbf{x}} \cdot\left(\mathbf{w}\left(t_{l}^{n}\right) u_{h}^{n+1}\right) \psi_{h} \mathrm{~d} \Omega+\frac{1}{2} \Delta t \sum_{l=0}^{m} \omega_{l} \int_{\Omega_{t_{l}-1}} \nabla_{\mathbf{x}} \cdot\left(\mathbf{w}\left(t_{l}^{n-1}\right) u_{h}^{n+1}\right) \psi_{h} \mathrm{~d} \Omega \\
& \quad=\Delta t \int_{\Omega_{t^{n+1}}} f^{n+1} \psi_{h} \mathrm{~d} \Omega \forall \psi_{h} \in \mathscr{X}_{0, h}\left(\Omega_{t}\right) .
\end{aligned}
$$


We will prove the following result:

Lemma 2. The discrete solution $u_{h}^{n}$ of scheme (36) satisfies the inequality

$$
\begin{aligned}
& \varepsilon_{h}^{n+1}+\Delta t \mu\left\|\nabla_{\mathbf{x}} u_{h}^{n+1}\right\|_{L_{2}\left(\Omega_{t^{n+1}}\right)}^{2}+\Delta t \sum_{l=0}^{m} \omega_{l} \int_{\Omega_{t_{l}^{n}}} \nabla_{\mathbf{x}} \cdot \mathbf{w}\left(t_{l}^{n}\right)\left|u_{h}^{n+1}\right|^{2} \mathrm{~d} \Omega \\
& \quad+\Delta t \sum_{l=0}^{m} \int_{\Omega_{t_{l}^{n-1}}} \nabla_{\mathbf{x}} \cdot \mathbf{w}\left(t_{l}^{n-1}\right)\left(\left|u_{h}^{n+1}\right|^{2}-2 u_{h}^{n+1} u_{h}^{n}\right) \mathrm{d} \Omega \leqslant \varepsilon_{h}^{n}+\Delta t \frac{\left(1+C_{\Omega}\right)}{\mu}\left\|f^{n+1}\right\|_{H^{-1}\left(\Omega_{t^{n+1}}\right)}^{2}
\end{aligned}
$$

for all $n=1,2, \ldots$, where

$$
\varepsilon_{h}^{n}=\frac{1}{2}\left\|u_{h}^{n}\right\|_{L_{2}\left(\Omega_{t^{n}}\right)}^{2}+\frac{1}{2}\left\|2 u_{h}^{n}-u_{h}^{n-1}\right\|_{L_{2}\left(\Omega_{t^{n-1}}\right)}^{2} .
$$

Proof. Let us take $\psi_{h}=u_{h}^{n+1}$ in (36). The first three terms can be developed in the following way

$$
\begin{aligned}
I= & \frac{3}{2} \int_{\Omega_{t^{n+1}}}\left|u_{h}^{n+1}\right|^{2} \mathrm{~d} \Omega-2 \int_{\Omega_{t^{n}}} u_{h}^{n} u_{h}^{n+1} \mathrm{~d} \Omega+\frac{1}{2} \int_{\Omega_{t^{n-1}}} u_{h}^{n-1} u_{h}^{n+1} \mathrm{~d} \Omega \\
= & \frac{3}{2} \int_{\Omega_{t^{n}}}\left|u_{h}^{n+1}\right|^{2} \mathrm{~d} \Omega-2 \int_{\Omega_{t^{n}}} u_{h}^{n} u_{h}^{n+1} \mathrm{~d} \Omega+\frac{1}{2} \int_{\Omega_{t^{n}}} u_{h}^{n-1} u_{h}^{n+1} \mathrm{~d} \Omega+\frac{3}{2} \Delta t \sum_{l=0}^{m} \omega_{l} \int_{\Omega_{t_{l}^{n}}} \nabla_{\mathbf{x}} \cdot \mathbf{w}\left(t_{l}^{n}\right)\left|u_{h}^{n+1}\right|^{2} \mathrm{~d} \Omega \\
& -\frac{1}{2} \Delta t \sum_{l=0}^{m} \omega_{l} \int_{\Omega_{t_{l}^{n-1}}} \nabla_{\mathbf{x}} \cdot \mathbf{w}\left(t_{l}^{n-1}\right) u_{h}^{n-1} u_{h}^{n+1} \mathrm{~d} \Omega
\end{aligned}
$$

where we have employed relation (29) with $v_{h}=u_{h}^{n+1}$ and $v_{h}^{2}=u_{h}^{n-1} u_{h}^{n+1}$. Let us observe, now, that

$$
\begin{aligned}
& \frac{3}{2} \int_{\Omega_{t^{n}}}\left|u_{h}^{n+1}\right|^{2} \mathrm{~d} \Omega-2 \int_{\Omega_{t^{n}}} u_{h}^{n} u_{h}^{n+1} \mathrm{~d} \Omega+\frac{1}{2} \int_{\Omega_{t^{n}}} u_{h}^{n-1} u_{h}^{n+1} \mathrm{~d} \Omega \\
& \quad=\frac{1}{2} \int_{\Omega_{t^{n}}}\left(u_{h}^{n+1}-2 u_{h}^{n}+u_{h}^{n-1}\right) u_{h}^{n+1} \mathrm{~d} \Omega+\frac{1}{2} \int_{\Omega_{t^{n}}}\left(2 u_{h}^{n+1}-u_{h}^{n}\right) 2 u_{h}^{n+1} \mathrm{~d} \Omega-\int_{\Omega_{t^{n}}}\left|u_{h}^{n+1}\right|^{2} \mathrm{~d} \Omega \\
& \quad=\frac{1}{4}\left\|u_{h}^{n+1}\right\|_{L_{2}\left(\Omega_{t^{n}}\right)}^{2}+\frac{1}{4}\left\|u_{h}^{n+1}-2 u_{h}^{n}+u_{h}^{n-1}\right\|_{L_{2}\left(\Omega_{t^{n}}\right)}^{2}-\frac{1}{4}\left\|2 u_{h}^{n}-u_{h}^{n-1}\right\|_{L_{2}\left(\Omega_{t^{n}}\right)}^{2} \\
& \quad+\frac{1}{4}\left\|2 u_{h}^{n+1}\right\|_{L_{2}\left(\Omega_{t^{n}}\right)}^{2}+\frac{1}{4}\left\|2 u_{h}^{n+1}-u_{h}^{n}\right\|_{L_{2}\left(\Omega_{t^{n}}\right)}^{2}-\frac{1}{4}\left\|u_{h}^{n}\right\|_{L_{2}\left(\Omega_{t^{n}}\right)}^{2}-\left\|u_{h}^{n+1}\right\|_{L_{2}\left(\Omega_{t^{n}}\right)}^{2} \\
& \quad=\left(\frac{1}{4}\left\|u_{h}^{n+1}\right\|_{L_{2}\left(\Omega_{t^{n}}\right)}^{2}+\frac{1}{4}\left\|2 u_{h}^{n+1}-u_{h}^{n}\right\|_{L_{2}\left(\Omega_{t^{n}}\right)}^{2}\right)+\frac{1}{4}\left\|u_{h}^{n+1}-2 u_{h}^{n}+u_{h}^{n-1}\right\|_{L_{2}\left(\Omega_{t^{n}}\right)}^{2} \\
& \quad-\left(\frac{1}{4}\left\|u_{h}^{n}\right\|_{L_{2}\left(\Omega_{t^{n}}\right)}^{2}+\frac{1}{4}\left\|2 u_{h}^{n}-u_{h}^{n-1}\right\|_{L_{2}\left(\Omega_{t^{n}}\right)}^{2}\right) .
\end{aligned}
$$

Then, applying (39) in (38) we obtain

$$
\begin{aligned}
I= & \frac{1}{4}\left\|u_{h}^{n+1}\right\|_{L_{2}\left(\Omega_{t^{n}}\right)}^{2}+\frac{1}{4}\left\|2 u_{h}^{n+1}-u_{h}^{n}\right\|_{L_{2}\left(\Omega_{t^{n}}\right)}^{2}+\frac{1}{4}\left\|u_{h}^{n+1}-2 u_{h}^{n}+u_{h}^{n-1}\right\|_{L_{2}\left(\Omega_{t^{n}}\right)}^{2}-\frac{1}{4}\left\|u_{h}^{n}\right\|_{L_{2}\left(\Omega_{t^{n}}\right)}^{2} \\
& -\frac{1}{4}\left\|2 u_{h}^{n}-u_{h}^{n-1}\right\|_{L_{2}\left(\Omega_{t^{n}}\right)}^{2}+\frac{3}{2} \Delta t \sum_{l=0}^{m} \omega_{l} \int_{\Omega_{t_{l}^{n}}} \nabla_{\mathbf{x}} \cdot \mathbf{w}\left(t_{l}^{n}\right)\left|u_{h}^{n+1}\right|^{2} \mathrm{~d} \Omega \\
& -\frac{1}{2} \Delta t \sum_{l=0}^{m} \omega_{l} \int_{\Omega_{t_{l}^{n-1}}} \nabla_{\mathbf{x}} \cdot \mathbf{w}\left(t_{l}^{n-1}\right) u_{h}^{n-1} u_{h}^{n+1} \mathrm{~d} \Omega
\end{aligned}
$$




$$
\begin{aligned}
= & \left(\frac{1}{4}\left\|u_{h}^{n+1}\right\|_{L_{2}\left(\Omega_{t^{n+1}}\right)}^{2}+\frac{1}{4}\left\|2 u_{h}^{n+1}-u_{h}^{n}\right\|_{L_{2}\left(\Omega_{t^{n}}\right)}^{2}\right)+\frac{1}{4}\left\|u_{h}^{n+1}-2 u_{h}^{n}+u_{h}^{n-1}\right\|_{L_{2}\left(\Omega_{t^{n-1}}\right)}^{2} \\
& -\left(\frac{1}{4}\left\|u_{h}^{n}\right\|_{L_{2}\left(\Omega_{t^{n}}\right)}^{2}+\frac{1}{4}\left\|2 u_{h}^{n}-u_{h}^{n-1}\right\|_{L_{2}\left(\Omega_{t^{n-1}}\right)}^{2}\right)+\frac{5}{4} \Delta t \sum_{l=0}^{m} \omega_{l} \int_{\Omega_{t_{l}^{n}}} \nabla_{\mathbf{x}} \cdot \mathbf{w}\left(t_{l}^{n}\right)\left|u_{h}^{n+1}\right|^{2} \mathrm{~d} \Omega \\
& +\frac{\Delta t}{4} \sum_{l=0}^{m} \omega_{l} \int_{\Omega_{t_{l}^{n-1}}} \nabla_{\mathbf{x}} \cdot \mathbf{w}\left(t_{l}^{n-1}\right)\left[\left(u_{h}^{n+1}-2 u_{h}^{n}+u_{h}^{n-1}\right)^{2}-\left(2 u_{h}^{n}-u_{h}^{n-1}\right)^{2}-2 u_{h}^{n-1} u_{h}^{n+1}\right] \mathrm{d} \Omega \\
= & \frac{1}{2}\left(\varepsilon_{h}^{n+1}-\varepsilon_{h}^{n}\right)+\frac{1}{4}\left\|u_{h}^{n+1}-2 u_{h}^{n}+u_{h}^{n-1}\right\|_{L_{2}\left(\Omega_{t^{n-1}}\right)}^{2}+\frac{5}{4} \Delta t \sum_{l=0}^{m} \omega_{l} \int_{\Omega_{t_{l}^{n}}} \nabla_{\mathbf{x}} \cdot \mathbf{w}\left(t_{l}^{n}\right)\left|u_{h}^{n+1}\right|^{2} \mathrm{~d} \Omega \\
& +\frac{1}{4} \Delta t \sum_{l=0}^{m} \omega_{l} \int_{\Omega_{t_{l}^{n-1}}} \nabla_{\mathbf{x}} \cdot \mathbf{w}\left(t_{l}^{n-1}\right)\left(\left|u_{h}^{n+1}\right|^{2}-4 u_{h}^{n+1} u_{h}^{n}\right) \mathrm{d} \Omega .
\end{aligned}
$$

Finally, by integrating by parts the convective terms in (36) and employing the previous result we have

$$
\begin{aligned}
& \frac{1}{2}\left(\varepsilon_{h}^{n+1}-\varepsilon_{h}^{n}\right)+\frac{1}{4}\left\|u_{h}^{n+1}-2 u_{h}^{n}+u_{h}^{n-1}\right\|_{L_{2}\left(\Omega_{t^{n-1}}\right)}^{2}+\frac{1}{2} \Delta t \sum_{l=0}^{m} \omega_{l} \int_{\Omega_{t_{l}^{n}}} \nabla_{\mathbf{x}} \cdot \mathbf{w}\left(t_{l}^{n}\right)\left|u_{h}^{n+1}\right|^{2} \mathrm{~d} \Omega \\
& \quad+\frac{1}{2} \Delta t \sum_{l=0}^{m} \omega_{l} \int_{\Omega_{t_{l}^{n-1}}} \nabla_{\mathbf{x}} \cdot \mathbf{w}\left(t_{l}^{n-1}\right)\left(\left|u_{h}^{n+1}\right|^{2}-2 u_{h}^{n+1} u_{h}^{n}\right) \mathrm{d} \Omega+\Delta t \mu\left\|\nabla_{\mathbf{x}} u_{h}^{n+1}\right\|_{L_{2}\left(\Omega_{t^{n+1}}\right)}^{2} \\
& \leqslant \Delta t \frac{\left(1+C_{\Omega}\right)}{2 \mu}\left\|f^{n+1}\right\|_{H^{-1}\left(\Omega_{t^{n+1}}\right)}^{2}+\Delta t \frac{\mu}{2}\left\|\nabla_{\mathbf{x}} u_{h}^{n+1}\right\|_{L_{2}\left(\Omega_{t^{n+1}}\right)}^{2}
\end{aligned}
$$

from which inequality (37) follows.

Should the domain be fixed, we would have the following global stability result

$$
\varepsilon_{h}^{n+1}+\frac{1}{2} \sum_{i=1}^{n}\left\|u_{h}^{i+1}-2 u_{h}^{i}+u_{h}^{i-1}\right\|_{L_{2}(\Omega)}^{2}+\Delta t \mu \sum_{i=1}^{n}\left\|\nabla_{\mathbf{x}} u_{h}^{i+1}\right\|_{L_{2}(\Omega)}^{2} \leqslant \varepsilon_{h}^{1}+\Delta t \frac{\left(1+C_{\Omega}\right)}{\mu} \sum_{i=1}^{n}\left\|f^{i+1}\right\|_{H^{-1}(\Omega)}^{2}
$$

without any condition on $\Delta t$.

In the case where the domain moves, we may recover a conditional stability result where the time step is bounded by quantities depending on the domain movement (more precisely on $\nabla_{\mathbf{x}} \cdot \mathbf{w}$ ). Again we refer to [10] for more details.

Remark 7. In a fixed domain problem, the quantity $\varepsilon_{h}^{n}$ turns out to be monotonically decreasing whenever $f=0$. Observe that $\varepsilon_{h}^{n} \rightarrow\left\|u_{h}\left(t^{n}\right)\right\|_{L_{2}(\Omega)}^{2}$ when $\Delta t \rightarrow 0$. From (41), we can also quantify the numerical dissipation of the scheme in the term $\frac{1}{2} \sum_{i=1}^{n}\left\|u_{h}^{i+1}-2 u_{h}^{i}+u_{h}^{i-1}\right\|_{L_{2}(\Omega)}^{2}$.

\section{Numerical assessment}

We have considered the model advection-diffusion problem (1) in a 2D domain. The ALE mapping has been constructed by solving a Laplace problem at each time step $t^{n}$ :

$$
\begin{cases}\Delta_{\mathbf{Y}} \mathscr{A}_{t^{n}}=0 & \text { in } \Omega_{0} \\ \mathscr{A}_{t^{n}}=\mathbf{Y}+\eta^{n} & \text { on } \partial \Omega_{0}\end{cases}
$$

$\eta^{n}$ being the displacement of the domain boundary at time $t^{n}$. 
We have considered both a piecewise linear and piecewise quadratic in time interpolations in each time slab $\left[t^{n}, t^{n+1}\right]$. The latter is obtained by interpolating the mappings $\mathscr{A}_{t^{n+1}}, \mathscr{A}_{t^{n}}$ and $\mathscr{A}_{t^{n-1}}$.

In both cases, we have taken $\mathbf{w}\left(Y, t^{n}\right)=\lim _{t \rightarrow t_{-}^{n}} \mathbf{w}(Y, t)$ since $\mathbf{w}$ is discontinuous at the time instants $t^{n}$.

All the numerical schemes proposed in the previous sections, i.e. the implicit Euler, the Crank-Nicolson and the $\mathrm{BDF}(2)$ schemes in both conservative and non-conservative formulation (in the following indicated by the suffixes $c$ and $n c$, respectively) have been implemented. In the non-conservative case, we have implemented the "standard version" of the aforementioned schemes since there is no need to modify them in order to satisfy the GCL. On the other hand, for the conservative formulation, we have considered the "standard" time discretization, which, in general, does not satisfy the GCL (in the following indicated with the suffix noGCL), the modified discretization that satisfies the GCL for a linear in time deformation of the domain (suffix GCL1) and the one that satisfies the GCL for a quadratic in time domain deformation (suffix GCL2). The quadrature formula $\mathscr{I}$ utilized is the mid-point rule for the schemes GCL1 and the two point Gaussian quadrature formula for the schemes GCL2. We remind that, for a 2D problem, the CrankNicolson scheme (28) always satisfies the GCL for a linear in time deformation of the domain. Then, in this case, scheme GCL0 coincides with GCL1.

We present hereafter two test cases. The first one aims at validating the stability results derived in the previous sections while the second one will focus on time accuracy.

\subsection{First test case-stability analysis}

We have taken as reference domain $\Omega_{0}$ the unit $2 \mathrm{D}$ square. The domain deformation is given by

$$
\mathbf{x}=\mathscr{A}_{t}(\mathbf{Y}):\left\{\begin{array}{l}
x_{1}=Y_{1}[2-\cos (20 \pi t)] \\
x_{2}=Y_{2}[2-\cos (20 \pi t)]
\end{array} .\right.
$$

We observe that the deformed domain is still a square that expands and contracts periodically with a period $T=1 / 10$. Moreover, since $\mathscr{A}_{t}(\mathbf{Y})$ is linear in $\mathbf{Y}$, by solving a Laplace problem at each time step $t^{n}$ for the discrete ALE mapping, we recover the deformation given in (43) exactly.

Yet, this deformation is interpolated polynomially in time in each time-slab. Thus, the numerical representation of the domain is not exact for $t \neq t^{n}, n=1,2, \ldots$

We have considered the problem

$$
\begin{cases}\frac{\partial u}{\partial t}-0.01 \Delta u=0, & \text { in } \Omega_{t} \\ u=0, & \text { on } \partial \Omega_{t} \\ u(0)=1600 Y_{1}\left(1-Y_{1}\right) Y_{2}\left(1-Y_{2}\right), & \text { in } \Omega_{0} .\end{cases}
$$

Observe that, as a consequence of the stability estimate (2), for such a problem $\|u(t)\|_{L_{2}\left(\Omega_{t}\right)}$ is a decreasing quantity.

Problem (44) has been discretized in space with $\mathbb{P}_{1}$ finite elements. The monotonicity property of the $L^{2}$ norm of the solution is clearly valid for the semi-discrete problem as well.

When problem (44) is defined on a fixed domain, the implicit-Euler and the Crank-Nicolson schemes preserve that property, i.e. the computed solution $u_{h}^{n}$ has a decreasing $L^{2}$ norm. On the other hand, when considering a moving domain, starting from our estimates, we should expect that only the implicit Euler scheme applied to the conservative formulation and satisfying the GCL will preserve that property.

Figs. 1 and 2 show, for the two schemes and the two cases of a linear and a quadratic interpolation of the domain deformation, the computed quantity $\left\|u_{h}^{n}\right\|_{L_{2}\left(\Omega_{t^{n}}\right)}^{2}$ together with the "exact" norm $\left\|u_{h}(t)\right\|_{L_{2}\left(\Omega_{t}\right)}^{2}$ of the solution of the semi-discrete problem (computed on the same mesh but with a very small time step) during 

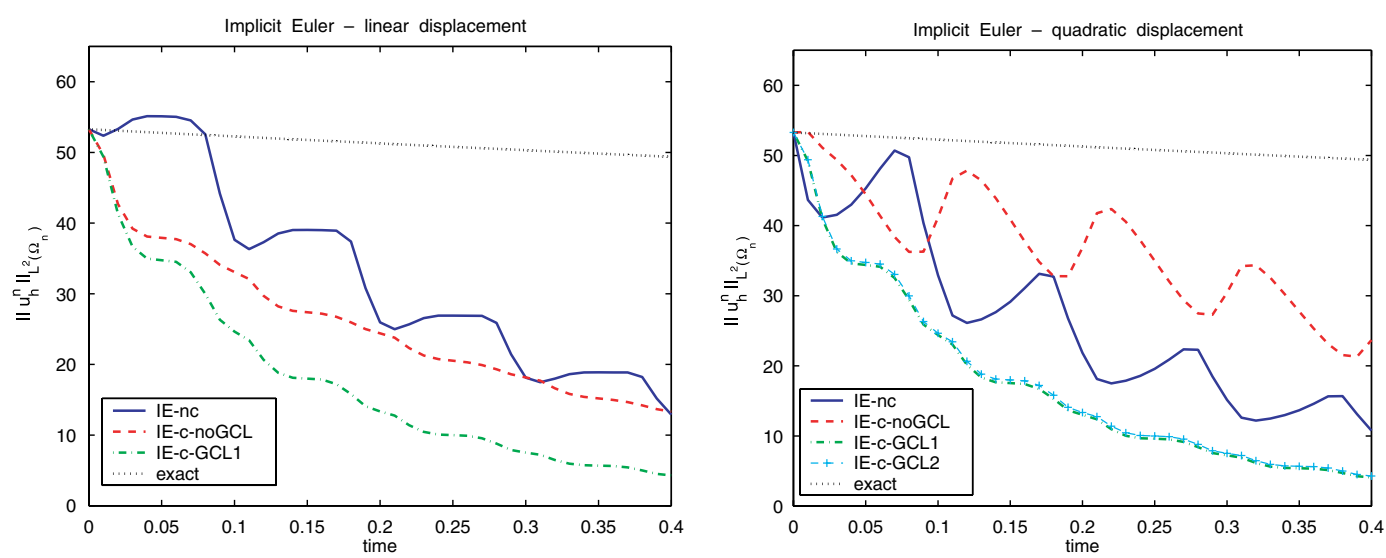

Fig. 1. Test case 1: $L^{2}$ norm of the computed and "exact" solution as a function of time for the implicit Euler scheme. Interpolation in time of the domain deformation: linear on the left and quadratic on the right.
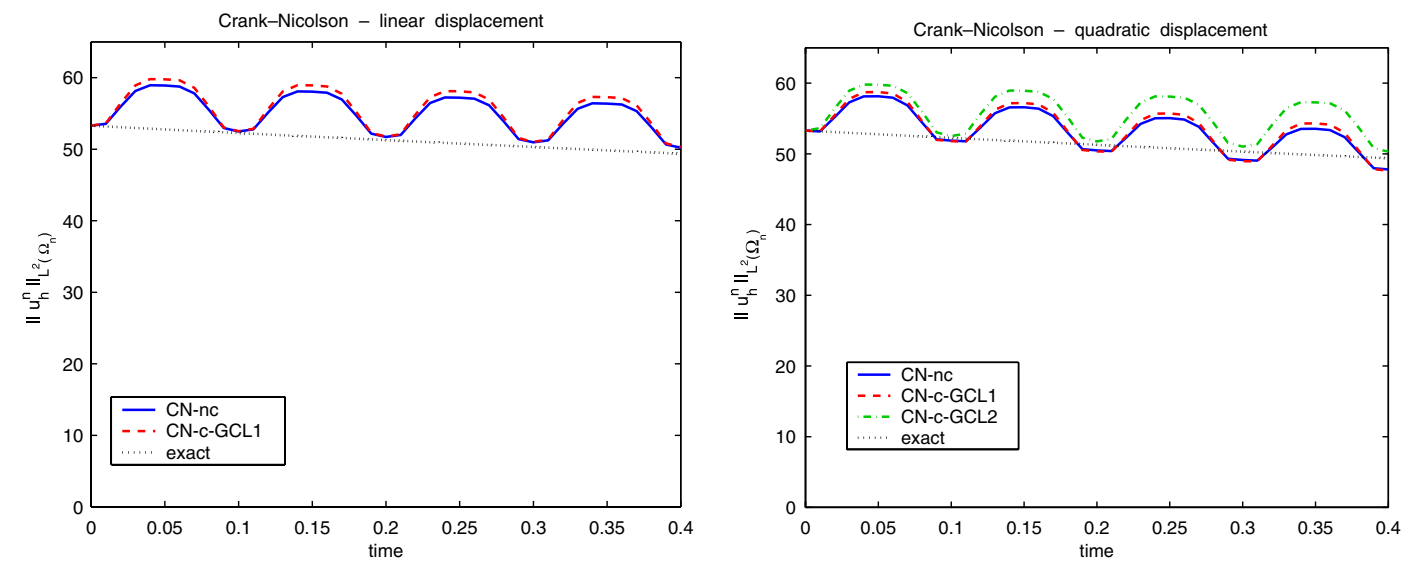

Fig. 2. Test case 1: $L^{2}$ norm of the computed and "exact" solution as a function of time for the Crank-Nicolson scheme. Interpolation in time of the domain deformation: linear on the left and quadratic on the right.

the time interval $[0,0.4]$ (corresponding to 4 periods of oscillation of the domain). In all cases we have used a time step $\Delta t=0.01$.

We can observe that, only the implicit Euler discretization which satisfies the GCL, applied to the conservative formulation, is strictly monotone, as it was predicted by our estimates.

Furthermore, for the Crank-Nicolson scheme, the $L^{2}$ norm increases during the expansion of the domain and decreases during the contraction phase, coherently with estimate (30).

The BDF(2) scheme, even when applied to problem (44) on a fixed domain, does not feature the monotonicity property of the $L^{2}$ norm of the solution. On the other hand, as shown by estimate (37), the quantity which turns out to be decreasing, for a problem on a fixed domain, is $\varepsilon_{h}^{n}=\frac{1}{2}\left\|u_{h}^{n}\right\|_{L_{2}\left(\Omega_{t^{n}}\right)}^{2}+\frac{1}{2} \| 2 u_{h}^{n}-$ $u_{h}^{n-1} \|_{L_{2}\left(\Omega_{t n-1}\right)}^{2}$ (see Remark 7).

Fig. 3 shows the quantity $\varepsilon_{h}^{n}$ for the different versions of the BDF(2) scheme and for the two cases of a linear and a quadratic in time interpolation of the domain deformation. On the same picture, we report also 

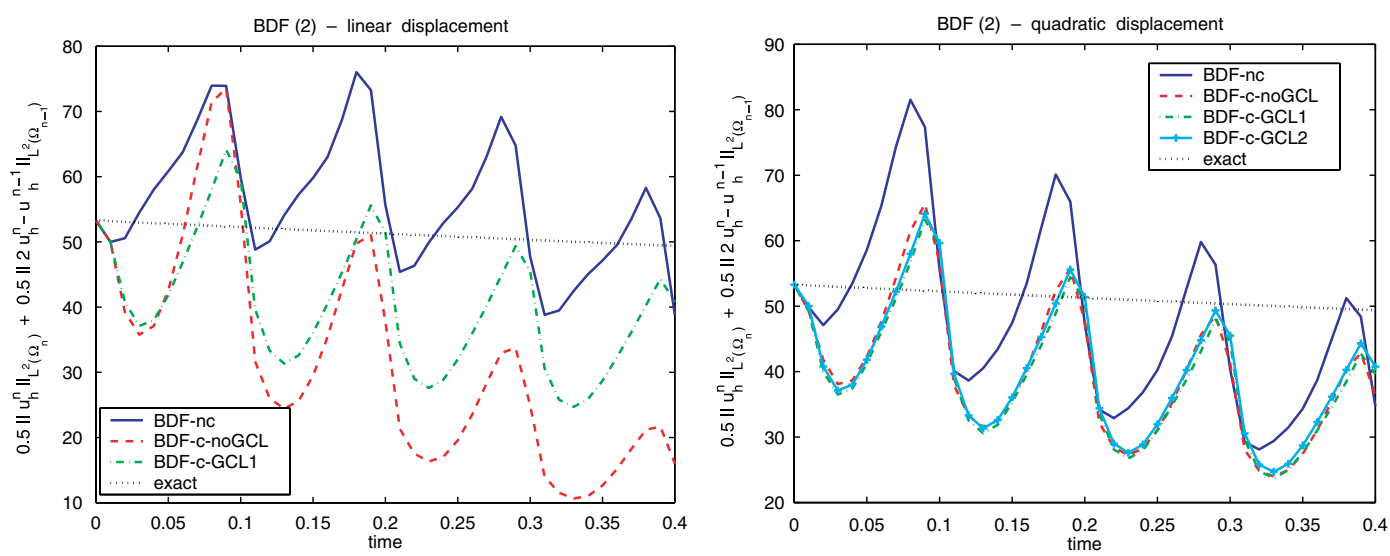

Fig. 3. Test case 1: quantity $\varepsilon_{h}^{n}$ as a function of time computed by the $\operatorname{BDF}(2)$ scheme, compared to the "exact" value $\left\|u_{h}(t)\right\|_{L_{2}\left(\Omega_{t}\right)}=\lim _{\Delta t \rightarrow 0} \varepsilon_{h}^{n}$. Interpolation in time of the domain deformation: linear on the left and quadratic on the right.

the norm $\left\|u_{h}(t)\right\|_{L_{2}\left(\Omega_{t}\right)}$ of the "exact" solution. It is evident that the quantity $\varepsilon_{h}^{n}$ is not decreasing, coherently with estimate (37).

Finally, in Fig. 4, we report the $L^{2}$ norm $\left\|u_{h}^{n}\right\|_{L_{2}\left(\Omega_{t^{n}}\right)}$ of the solution computed by the BDF(2) scheme. This figure highlights the dissipation properties of this scheme and should be compared to Figs. 1 and 2.

\subsection{Second test case-error analysis in time}

We have considered again as reference configuration $\Omega_{0}$ the unit $2 \mathrm{D}$ square. The domain deformation is given by

$$
\mathbf{x}=\mathscr{A}_{t}(\mathbf{Y}):\left\{\begin{array}{l}
x_{1}=Y_{1}[2-\cos (10 \pi t)] \\
x_{2}=Y_{2}[2-\cos (10 \pi t)]
\end{array} .\right.
$$
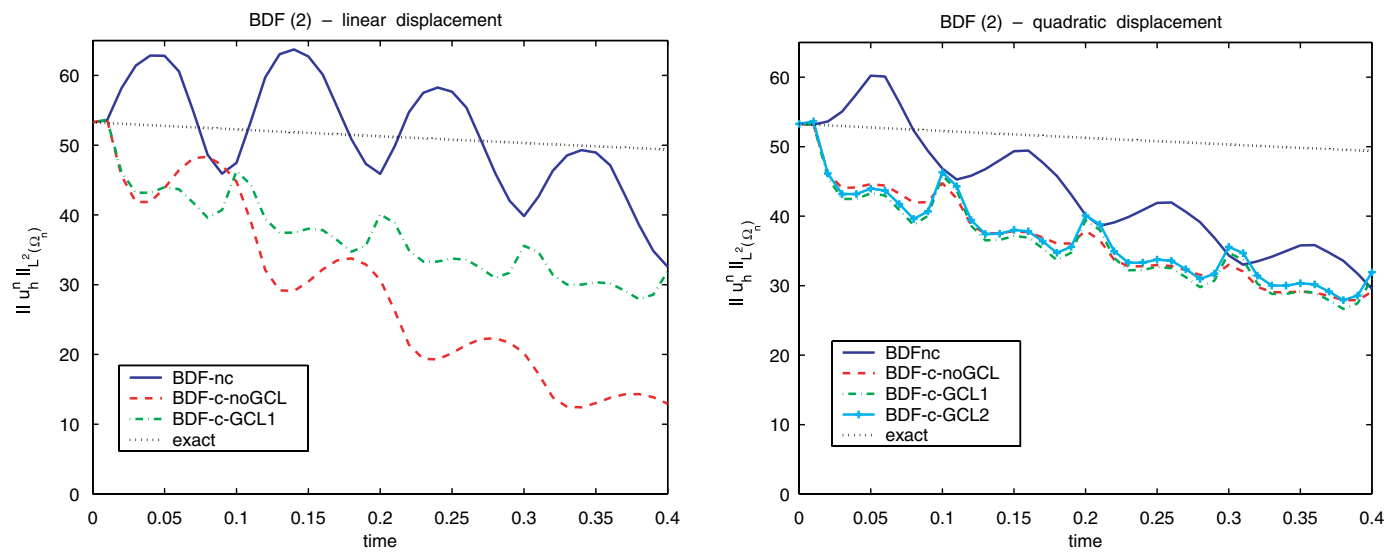

Fig. 4. Test case 1: $L^{2}$ norm of the computed and "exact" solution as a function of time for the BDF(2) scheme. Interpolation in time of the domain deformation: linear on the left and quadratic on the right. 

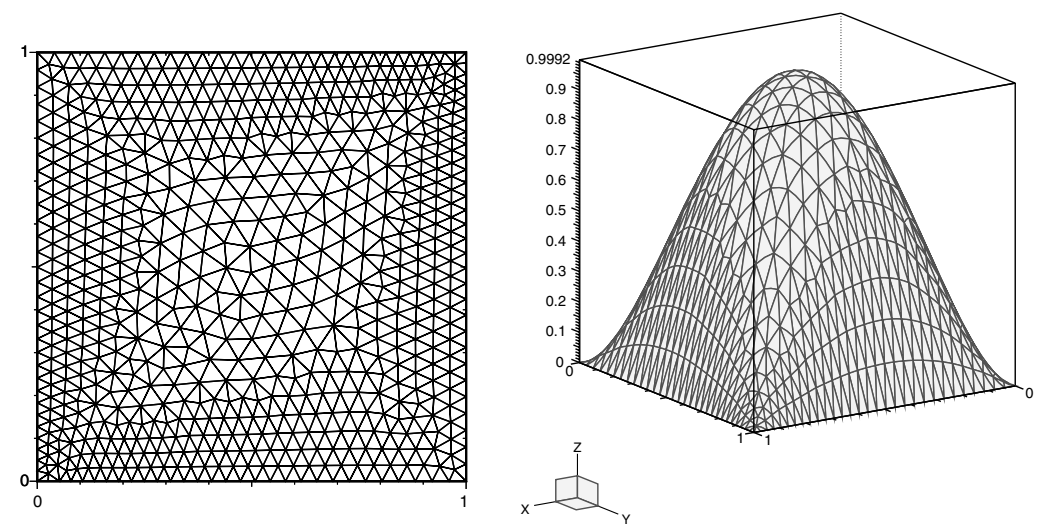

Fig. 5. Mesh and initial solution of the problem illustrated in test case 3 .

The problem we have considered is

$$
\begin{cases}\frac{\partial u}{\partial t}-0.1 \Delta u=f, & \text { in } \Omega_{t} \\ u=0, & \text { on } \partial \Omega_{t} \\ u(0)=16 Y_{1}\left(1-Y_{1}\right) Y_{2}\left(1-Y_{2}\right), & \text { in } \Omega_{0} .\end{cases}
$$

The forcing term $f$ has been chosen in such a way that the corresponding exact solution $u(\mathbf{Y}, t)$ is

$$
u(\mathbf{Y}, t)=16\left(1+\frac{1}{2} \sin (5 \pi t)\right) Y_{1}\left(1-Y_{1}\right) Y_{2}\left(1-Y_{2}\right) .
$$

Problem (46) has been discretized in space with $\mathbb{P}_{2}$ isoparametric elements. Fig. 5 shows the mesh used (on the left) and the initial solution (on the right).

We have taken a sequence of decreasing time steps $\Delta t=1 / 20,1 / 40, \ldots, 1 / 320$ and we have computed the $L^{2}$ norm of the error at time $t=0.3$ over the actual domain $\Omega_{t}$. In all cases the error is dominated by the time discretization. The results obtained are presented in Figs. 6-8 for the implicit Euler, the CrankNicolson and the BDF(2) schemes, respectively.

We observe that all the different implementations of the implicit Euler scheme are linearly convergent in time while all the Crank-Nicolson ones are quadratically convergent.
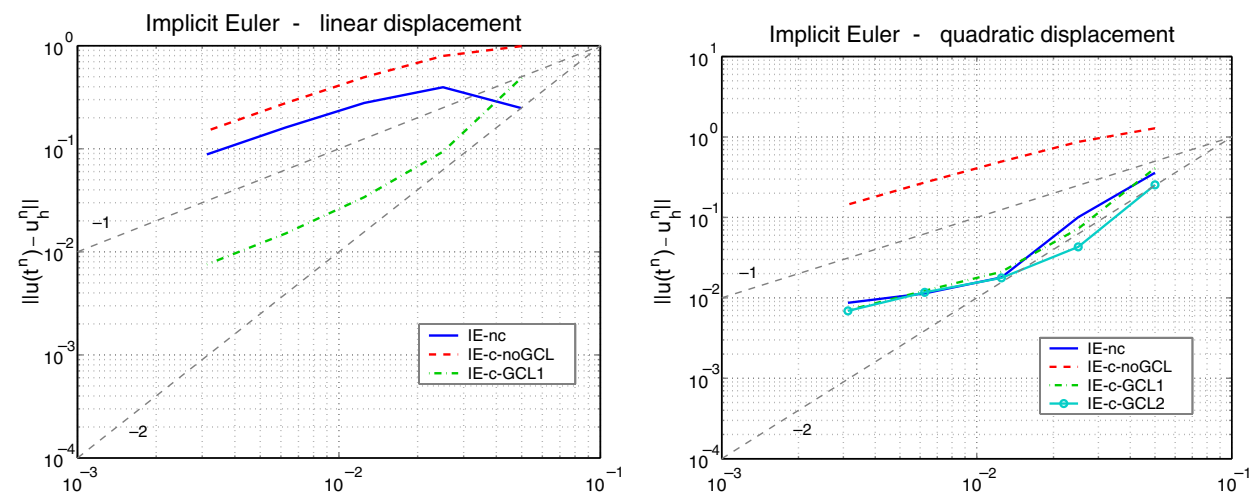

Fig. 6. Test case 3: error in the $L^{2}$ norm as a function of the time step $\Delta t$ for the implicit Euler scheme. Interpolation in time of the domain deformation: linear on the left and quadratic on the right. 

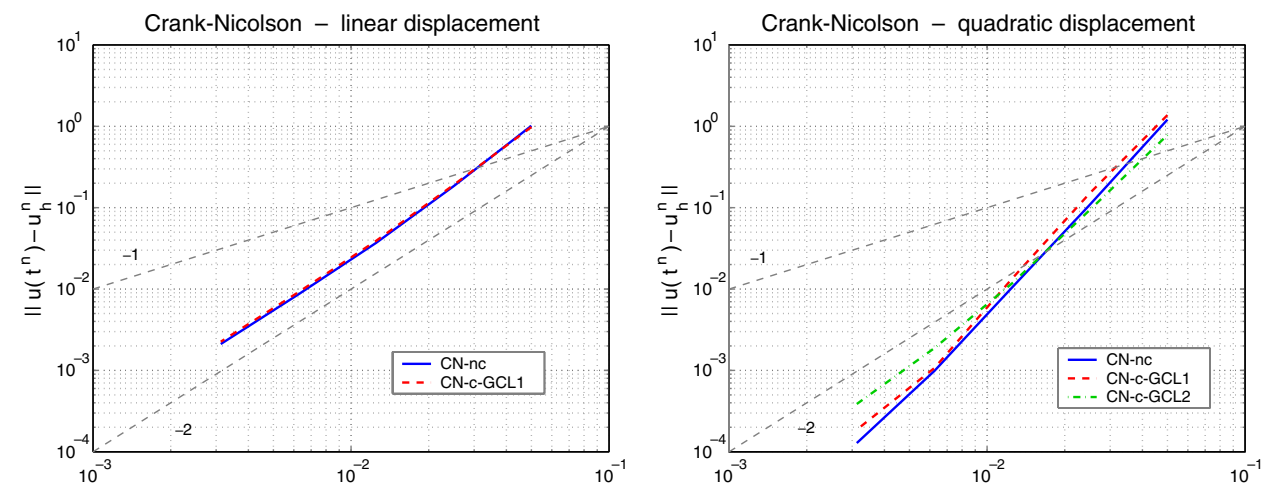

Fig. 7. Test case 3: error in the $L^{2}$ norm as a function of the time step $\Delta t$ for the Crank-Nicolson scheme. Interpolation in time of the domain deformation: linear on the left and quadratic on the right.
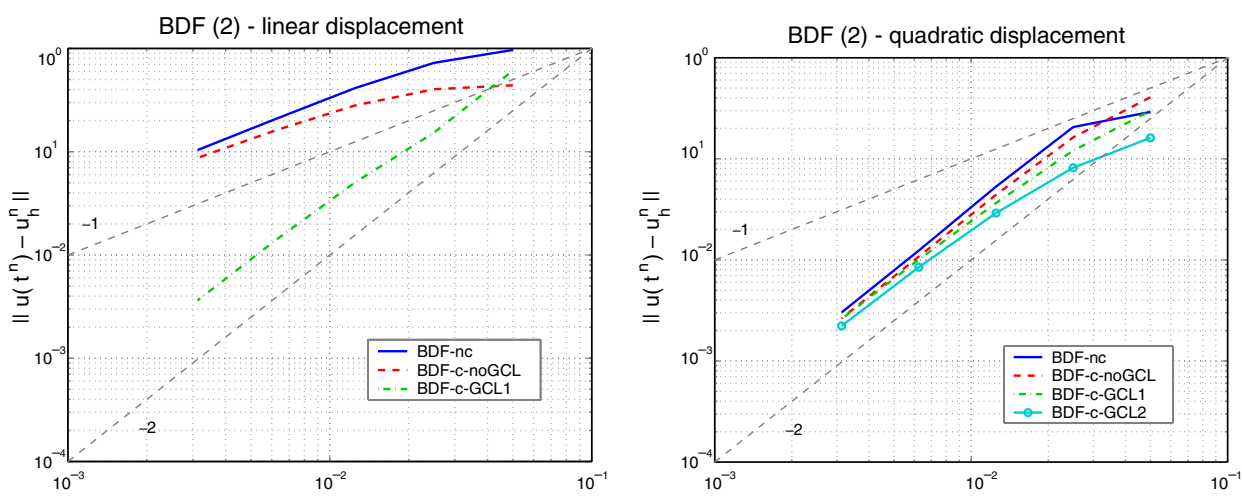

Fig. 8. Test case 3: error in the $L^{2}$ norm as a function of the time step $\Delta t$ for the BDF(2). Interpolation in time of the domain deformation: linear on the left and quadratic on the right.

On the contrary, the $\mathrm{BDF}(2)$ scheme, applied to the non-conservative formulation or to the conservative one, without satisfying the GCL, is only linearly convergent when a linear in time interpolation of the domain deformation is considered. We recover a second-order accuracy when employing a quadratic interpolation of the domain deformation.

Finally, we remark that the $\operatorname{BDF}(2)$ scheme that satisfies the GCL, applied to the conservative formulation, preserves the second-order accuracy even though the domain deformation is only linearly interpolated in time.

\section{Conclusions}

This work shows how a careful choice of the time integration formula and mesh movement/velocity reconstruction allows to maintain a high order time accuracy of a numerical scheme applied to a moving domain problem.

However it also shows the difficulties, for the discrete problem, in maintaining unconditional stability in the moving mesh case, irrespectively on the domain velocity. This is a potential problem since in 
fluid-structure interaction the boundary velocity (and consequently the domain velocity reconstructed through the ALE map) is itself an unknown. In non-linear problems, this difficulties may be even amplified by non-linear instability issues.

Another finding is that the satisfaction of the GCL is neither a necessary nor a sufficient condition for stability, apart from special cases like the backward Euler scheme. This in principle could reduce the interest in GCL-satisfying schemes. However, we may say, also in view of the results shown in [3] that satisfying the GCL might help in improving the accuracy of the scheme and, in special cases, also to enhance stability. Therefore, in particular for fluid structure problems, it might be a good idea to stick to GCL-satisfying schemes.

Part of the analysis illustrated in this work has been extended to fluid-structure interaction problems, where the fluid is modeled by the incompressible Navier-Stokes equation [10] and has not been reported here for the sake of brevity.

\section{References}

[1] R. Aris, Vectors, Tensors, and the Basic Equations of Fluid Mechanics, Dover Publications Inc., 1989.

[2] H. Brezis, Analyse Fonctionnelle, Théorie et Applications, Masson, 1983.

[3] C. Fahrat, P. Geuzaine, C. Grandmont, The discrete geometric conservation law and the non-linear stability of ALE schemes for the solution of flow problems on moving grids, J. Comp. Phys. 174 (2001) 669-692.

[4] L. Formaggia, F. Nobile, A stability analysis for the Arbitrary Lagrangian Eulerian formulation with finite elements, East-West J. Num. Math. 7 (1999) 105-132.

[5] P. Geuzaine, C. Grandmont, C. Fahrat, Design and analysis of ALE schemes with provable second-order time-accuracy for inviscid and viscous flow simulations, J. Comp. Phys. 191 (2003) 206-227.

[6] H. Guillard, C. Farhat, On the significance of the geometric conservation law for flow computations on moving meshes, Comput. Methods Appl. Mech. Engrg. 190 (2000) 1467-1482.

[7] B. Koobus, C. Farhat, Second-order time-accurate and geometrically conservative implicit schemes for flow computations on unstructured dynamic meshes, Comput. Methods Appl. Mech. Engrg. 170 (1999) 103-129.

[8] M. Lesoinne, C. Farhat, Geometric conservation laws for aeroelastic computations using unstructured dynamics meshes, AIAA95-1709, Presented at the 12th AIAA Computational Fluid Dynamics Conference, San Diego, June 1995.

[9] M. Lesoinne, C. Farhat, Geometric conservation laws for flow problems with moving boundaries and deformable meshes, and their impact in aeroelastic computations, Comput. Methods Appl. Mech. Engrg. 134 (1996) 71-90.

[10] F. Nobile, Numerical approximation of fluid-structure interaction problems with application to haemodynamics, Ph.D. thesis, École Polytechnique Fédérale de Lausanne, 2001. Thesis No. 2458.

[11] A. Quarteroni, A. Valli, Numerical Approximation of Partial Differential Equations, Springer Verlag, 1994.

[12] P.D. Thomas, C.K. Lombard, Geometric conservation law and its application to flow computations on moving grids, AIAA J. 17 (1979) 1030-1037.

[13] H. Zhang, M. Reggio, J.Y. Trèpanier, R. Camarero, Geometric form of the GCL for moving meshes and its implementation in CFD schemes, Comput. Fluids 22 (1) (1993) 9-23. 